\title{
Analyses of the method development of decisions in an expert system with the use of information from an artificial neural network
}

\author{
Stanisław Duer ${ }^{1,}$, Konrad Zajkowski ${ }^{1}$, Serghei Scaticailov ${ }^{2}$ and Pawet Wrzesien ${ }^{3}$ \\ ${ }^{1}$ Koszalin University of Technology, Department of Mechanical Engineering, Poland \\ ${ }^{2}$ Technical University of Moldova, Department of Manufacturing Engineering, Republic of Moldova \\ ${ }^{3}$ vortex energy Polska sp. z o.o., Department of Technical and Commercial Management, Poland
}

\begin{abstract}
The present article covers the use of an artificial intelligence system in the organization of the prevention of technical objects. For this purpose, the composition of this system including an intelligent diagnostic system and an intelligent maintenance system was characterized and described. An artificial neural network and an expert system, which work among others on the basis of the information developed by the neural network, perform a special function in these systems. It was mentioned in the article that the mathematical model of the regeneration process of the functional properties (prevention) of an object forms the basis of the organization of the prevention activities of technical devices and objects with the use of intelligent systems. This model demonstrated the possibilities and directions for the use of maintenance intelligent systems.
\end{abstract}

\section{Introduction}

In technical and production systems and other fields of life, artificial intelligence methods have a wide range of applications in solving of various complex problems. Intelligent systems constitute modern solutions which use artificial neural networks, expert systems, genetic and evolutionary algorithms with the use of various collections of knowledge including fuzzy sets. Neural networks possess an ability of approximation, learning on the grounds of information (knowledge) from the sets of numerical data; they also possess an ability to generalize knowledge collected and an ability to build new (current) models of the system on the grounds of input and output data taking into consideration inexplicit relations between the data. Fuzzy logic is characterized by an ability to process fuzzy knowledge through inference on the grounds of explicitly defined linguistic rules. Therefore, this makes it possible to take decisions in the case when there are no explicitly defined mathematical models. Evolutionary algorithms are characterized by an ability of adaptation and self-learning, and they enable an effective search of the alternative spaces of solutions in order to find the best (optimal) solutions.

The purpose of artificial intelligence in technical applications is the realization of selected functions of the human mind and behaviour, including reaction, activities, senses,

*Corresponding author: stanislaw.duer@tu.koszalin.pl 
behaviours, taking decisions etc., which cannot be subjected to a simple numerical algorithmization. Therefore, it constitutes an effective tool for logical and rational inference, and for taking decisions in such conditions where incomplete data is present. When applying artificial intelligence methods, it is not only data but also knowledge that is processed in production processes. For this reason, a technical and production system can be operated with the use of explicit and inexplicit knowledge. An approach which consists in procedural processing based on algorithms is replaced with intelligent searching in technological systems through the applications of artificial intelligence methods. This makes it possible to create intelligent systems to support decisions in technological process [1-3, 3, 8, 13-17].

\section{Examination of the development of decisions in the process creation of an expert servicing knowledge of the radar system}

As a result, the maintenance system produces a set of maintenance information $\left\{M_{E}\left(e_{i, j}\right)\right\}$, which will be used for organization of the objects, which is presented in Table 1.

Table 1. Structure of the servicing maintenance information of the object.

\begin{tabular}{|c|c|c|c|c|c|c|}
\hline \multirow[t]{2}{*}{ Object } & \multirow{2}{*}{$\begin{array}{c}\text { Servicing } \\
\text { levels } \\
\text { of object }\end{array}$} & \multicolumn{5}{|c|}{$\begin{array}{l}\text { Vector of the servicing maintenance } \\
\text { information of the object }\left[M_{E}\left(e_{i, j}\right)\right]\end{array}$} \\
\hline & & $M_{E}\left(e_{1,1}\right)$ & $\ldots$ & $M_{E}\left(e_{i, j}\right)$ & $\ldots$ & $M_{E}\left(e_{i, J}\right)$ \\
\hline \multirow{6}{*}{$\mathrm{O}$} & 1 & $M_{E}\left(e_{1,1}\right)$ & $\ldots$ & $M_{E}\left(e_{1, \mathrm{j}}\right)$ & $\ldots$ & $M_{E}\left(e_{1, J}\right)$ \\
\hline & $\vdots$ & $\vdots$ & $\ldots$ & $\vdots$ & $\ldots$ & $\vdots$ \\
\hline & & & & & & \\
\hline & $i$ & $M_{E}\left(e_{i, 1}\right)$ & $\ldots$ & $M_{E}\left(e_{i, j}\right)$ & $\ldots$ & $M_{E}\left(e_{i, J}\right)$ \\
\hline & $\vdots$ & $\vdots$ & $\ldots$ & $\vdots$ & $\ldots$ & $\vdots$ \\
\hline & $\bar{I}$ & $M_{E}\left(e_{I, 1}\right)$ & $\ldots$ & $M_{E}\left(e_{I, j}\right)$ & $\ldots$ & $\overline{M_{E}\left(e_{I, J}\right)}$ \\
\hline
\end{tabular}

Where: $M_{E}\left(e_{i, j}\right)$ - servicing maintenance information of $j^{\text {th }}$ element in $i^{\text {th }}$ assembly.

The final form of the maintenance knowledge base was presented in the form of the information included in Table 1. This table includes pieces of maintenance information (rules) for the object. Each rule included in this table determines explicitly which element of a given assembly of the object must be subject to regeneration, and what range of prevention activities (from the set of the activities) is to be performed on it. The execution of the determined set of maintenance rules will result in the regeneration of the whole element, assembly and the whole object [1-3, 8-12]. The technical object, once the maintenance has been performed, is subject to a control check-up (testing) of the state, and in the case of a negative result of the control, the object is once again referred to prevention. The examination of the quality of the regeneration of an object in a maintenance system organized on the basis of maintenance information was conducted for the three following manners of expert inference:

1. Deductive

2. Probabilistic

3. Inductive.

The core of the qualitative process of the preventive procedures of a technical object involves a two-stage examination. In the first stage, a regeneration (preventive procedures) of the object is conducted on the basis of the maintenance information which is determined with the use of a given expert inference method. It is only in the second stage that the quality of these preventive procedures is assessed on the basis of control diagnosing of the object after the preventive procedures have been conducted. The diagnosing of a control object is performed in a diagnostic system with the use of the (DIAG) neural network [4-7]. It was assumed in the experimental examination of the preventive 
procedures of the object that each of the three inference methods has an influence on the preventive procedures in such a way that it guarantees a full regeneration of the object.

The first method of the object's preventive procedures (model I) is performed with the use of a deductive manner of expert inference. From there, the testing vector was developed of the maintenance information being determined for the deductive method of expert inference (model I), which accepts the following form (1):

$$
\left\{M_{E}\left(e_{i, j}\right)_{I}\right\}=\left\lfloor M_{E}\left(e_{1,1}\right)_{I}, \ldots, M_{E}\left(e_{i, j}\right)_{I}, \ldots, M_{E}\left(e_{I, J}\right)_{I}\right\rfloor
$$

Where: $M_{E}\left(e_{i, j}\right) \mathrm{I}-$ servicing maintenance information of $j^{\text {th }}$ element in $i^{\text {th }}$ assembly for a model $I$.

On the basis of this information: dependence (1), the maintenance system was organized (Figure 2), in which the technical object was regenerated. Once the preventive procedures have been performed, the technical object undergoes control diagnosing with the use of the diagnostic artificial neural network in a (DIAG) computer program. The results obtained of the diagnosing of the object (for the model I) were presented in the form of Figure 1.

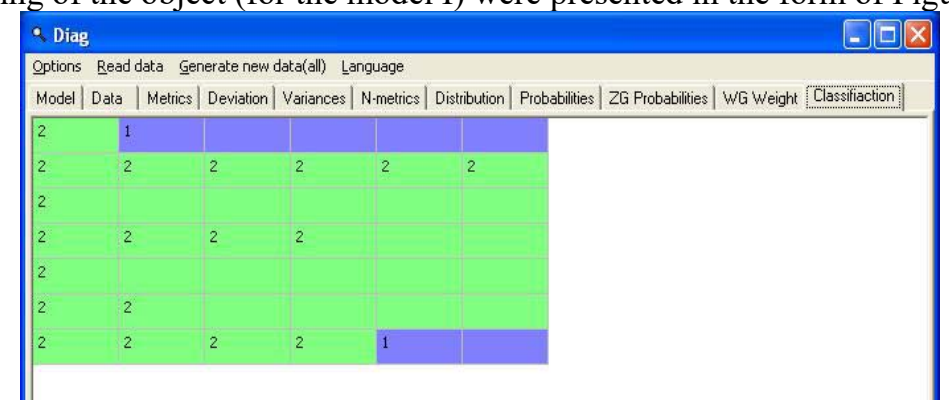

Fig. 1. The final results obtained in the DIAG diagnostic programme - "TABLE OF STATES OF THE OBJECT" (model I).

Where: "2" - the up state, "1" - the incomplete up state, " 0 " - the down state.

Further, similarly as described above, the maintenance was performed of the technical object for the inductive method of expert inference. The preventive procedures of the object were performed with the use of the testing vector of maintenance information that was determined for the inductive method of expert inference (a model II), and which was presented in the following analytical form (2):

$$
\left\{M_{E}\left(e_{i, j}\right)_{I I}\right\}=\left\lfloor M_{E}\left(e_{1,1}\right)_{I I}, \ldots, M_{E}\left(e_{i, j}\right)_{I I}, \ldots, M_{E}\left(e_{I, J}\right)_{I I}\right\rfloor
$$

Where: $M_{E}\left(e_{i, j}\right)$ II - servicing maintenance information of $j^{\text {th }}$ element in $i^{\text {th }}$ assembly for (model II).

After the preventive procedures have been performed, the technical object, as in the previous case, underwent control diagnosing with the use of the (DIAG) computer program. The results obtained of the diagnosing of the object for the model II were presented in the form of Figure 2.

In the further example of the experiment, the influence was tested of the probabilistic method of expert inference on the regeneration quality of the object in an organized maintenance system. 


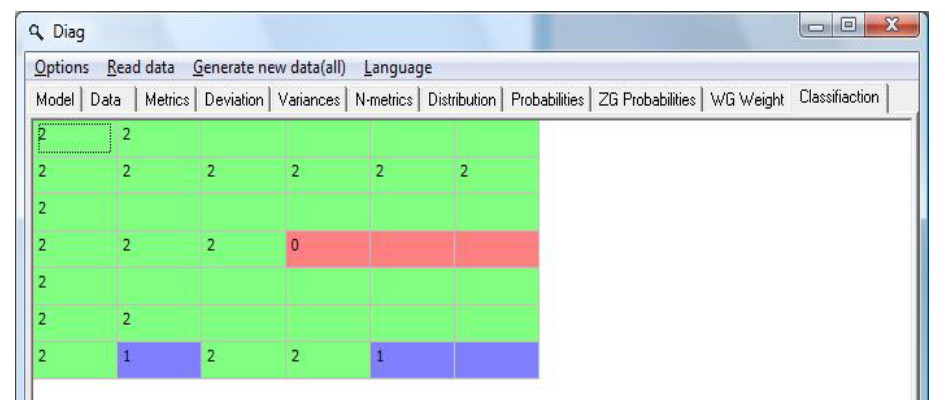

Fig. 2. The final results obtained in the (DIAG) diagnostic programme - "TABLE OF STATES OF THE OBJECT" (model II).

Where: "2" - the up state, "1" - the incomplete up state, " 0 " - the down state.

For this method of the expert's work, a set of maintenance information was determined, which was set up in the form of the testing vector of the maintenance information set. The maintenance information set that was determined for the probabilistic method of expert inference (model III) accepts the form of the following dependence (3):

$$
\left\{M_{E}\left(e_{i, j}\right)_{I I I}\right\}=\left\lfloor M_{E}\left(e_{1,1}\right)_{I I I}, \ldots, M_{E}\left(e_{i, j}\right)_{I I I}, \ldots, M_{E}\left(e_{I, J}\right)_{I I I}\right\rfloor
$$

Where: $M_{E}\left(e_{i, j}\right)$ III - servicing information of $j^{\text {th }}$ element in $i^{\text {th }}$ assembly for model II.

Once the preventive procedures have been performed, the technical object undergoes control diagnosing (examination) with the use of the diagnostic neural network in (DIAG) program. The results obtained of the diagnosing of the object (for model III) were presented in the form of (Figure 3).

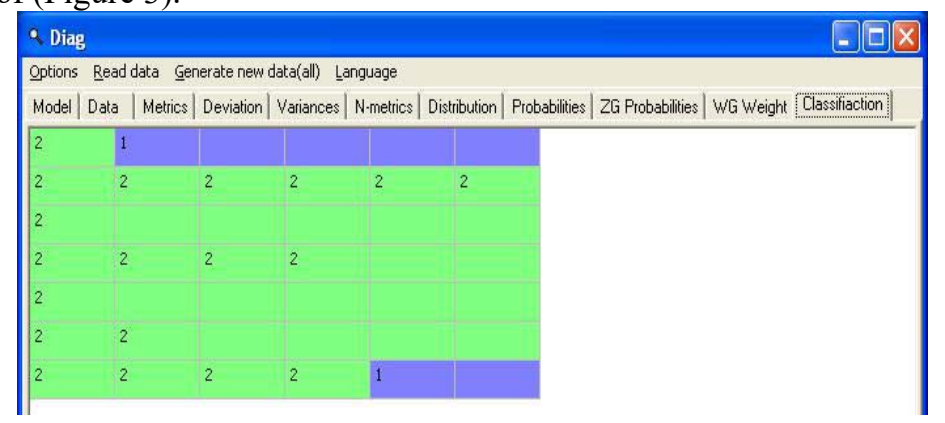

Fig. 3. The final results obtained of (DIAG) diagnostic programme - "TABLE OF STATES OF THE OBJECT" (model III).

Where: "2" - the up state, "1" - the incomplete up state, " 0 " - the down state.

A function of the qualitative evaluation of the maintenance process of the object was developed.

Definition: The qualitative function of the control qualitative evaluation of the preventive procedures process of a technical object $\left(F_{r}\right)$ in a maintenance system is the quantity which determines the set of fully regenerated functional elements of a technical object in relation to the set of all those elements of the object which have undergone a maintenance process. This quantity was presented in the form of dependence (4):

$$
F_{r}=\frac{n-k}{n} \cdot 100 \%
$$


Where: $n$ - the set of all those elements of the object which underwent the maintenance process - a set of those elements which determine the object's maintenance structure $\left\{e_{i, j}\right\}$, $k$ - a set of the fully regenerated (with the fitness states being recognized) primary elements of the object.

The results obtained from the examination in the experiment were the subject of an analysis and a study with the use of dependence (4) and Figure 4. The quantities obtained are graphically presented in (Figure 4).

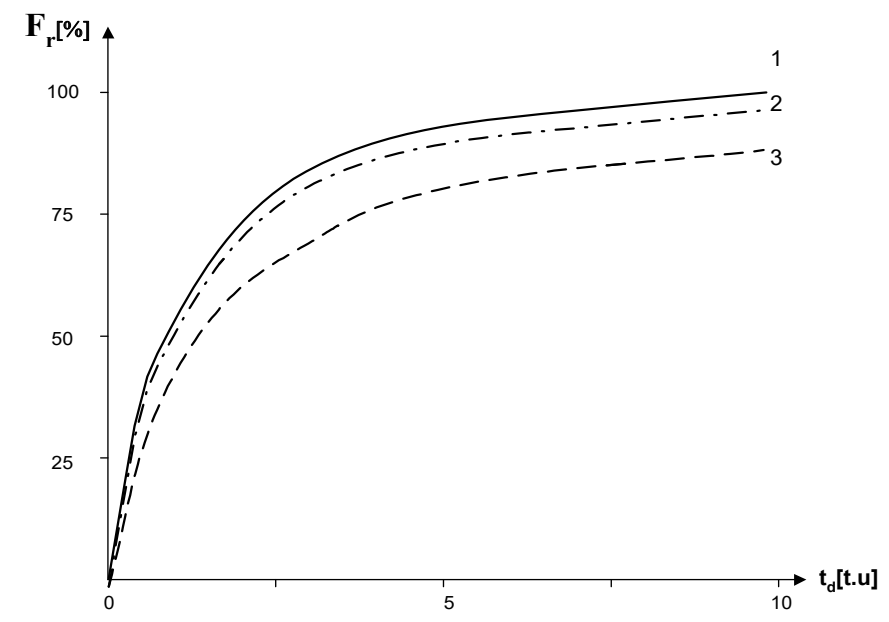

Fig. 4. Diagram of the qualitative function of the regeneration of the elements of a technical object $\left(\mathrm{F}_{\mathrm{r}}\right)$ by the maintenance system.

Where: 1 - the diagram for the vector of the testing maintenance information (model I); 2 the diagram for the vector of the testing maintenance information (model III); 3 - the diagram for the vector of the testing maintenance information (model II); $t_{d}\left(e_{i, j}\right)-$ the regeneration time of the $j^{\text {th }}$ primary element in the $i^{\text {th }}$ functional units of the object, $t . u-$ is time of unit.

If the time is known of the diagnosis (recognition of the states) of the individual elements of the object, then the function can be established of the qualitative regeneration of the object in the maintenance system $\left(F_{r}=f\left(t_{d}\left(e_{i, j}\right)\right)\right.$ or $\left.F_{n r}=f\left(t_{d}\left(e_{i, j}\right)\right)\right)$, where: $\left(t_{d}\right)$ is the diagnosing time of the states of a technical object for $\left(j^{\text {th }}\right)$ element in $\left(i^{\text {th }}\right)$ unit of the object $\left(e_{i, j}\right)$.

\section{Conclusions}

The material presented in this paper demonstrates that the prevention of technical objects constitutes a difficult organizational and technical undertaking. On the one hand, the abovementioned difficulty consists in obtaining reliable diagnostic information concerning the object (an artificial neural network was applied here). On the other hand, there is a problem connected with the transformation of diagnostic information into the form of maintenance information (an expert system was applied here). The transformation of diagnostic information into the required form which can be used takes place according to the algorithm presented. In this process, the specialist knowledge of a human specialist is used, as well. Hence, an adequate and proper taking of decisions is essential. Two inference methods were characterized and described in this paper. Each of them has its advantages and disadvantages. The influence of each of these inference methods on the final results of 
the maintenance process, which is the quality of the results obtained, was presented in this study.

\section{References}

1. R.E. Barlow, F. Proschan. Mathematical Theory of Reliability (J.Wiley \& Sons, New York 1995)

2. L. Będkowski, T. Dąbrowski. The Basis of Exploitation, Part II: The Basis of Exploational Reliability (Military University of Technology, Poland, 2006)

3. A. Birolini, Reliability Engineering Theory and Practice (Springer, New York, 1999)

4. B. Buchannan, E. Shortliffe. Rule - Based expert systems (Addison - Wesley Publishing Company, 1985)

5. S. Duer, Diagnostic system for the diagnosis of a reparable technical object, with the use of an artificial neural network of RBF type. Neural Computing \& Applications 19(5), 691-700 (2010)

6. S. Duer, R. Duer. Diagnostic system with an artificial neural network which determines a diagnostic information for the servicing of a reparable technical object. Neural Computing \& Applications 19(5), 755-766 (2010)

7. S. Duer Artificial neural network in the control process of object's states basis for organization of a servicing system of a technical objects. Neural Computing \& Applications, 21(1), 153-160 (2012)

8. S. Duer, K. Zajkowski. Taking decisions in the expert intelligent system to support maintenance of a technical object on the basis information from an artificial neural network. Neural Computing \& Applications 23(7), 2185-2197 (2013)

9. S. Duer, K. Zajkowski, R. Duer, J. Paś. Designing of an effective structure of system for the maintenance of a technical object with the using information from an artificial neural network. Neural Computing \& Applications, 23(3-4), 913-925 (2013)

10. S. Duer, Applications of an artificial intelligence for servicing of a technical object. Neural Computing \& Applications 22(5), 955-968 (2013)

11. S. Duer, D. Bernatowicz. The computer diagnostic program (DIAG 2) for identifying states of complex technical objects. E3S Web of Conferences, 19, 01029 (2017)

12. M. Madan, M. Gupta, J. Liang, N. Homma, Static and Dynamic Neural Networks, From Fundamentals to Advanced Theory (John Wiley \& Sons, Inc, Hoboken, New Jersey, 2003)

13. T. Nakagawa. Maintenance Theory of Reliability (Springer - Verlag London Limited, 2005)

14. T. Nakagawa, K. Ito. Optimal inspection policies for a storage system with degradation at periodic tests. Math. Comput. Model 31, 191-195 (2000)

15. K. Zajkowski. The method of solution of equations with coefficients that contain measurement errors, using artificial neural network. Neural Computing and Applications 24(2), 431-439 (2014)

16. K. Zajkowski. An innovative hybrid insulation switch to enable/disable electrical loads without overvoltages. E3S Web of Conferences 19, 01033 (2017)

17. K. Zajkowski. Settlement of reactive power compensation in the light of white certificates. E3S Web of Conferences 19, 01037 (2017) 